\title{
Surveillance of Human Papilloma Virus Using Reference Laboratory Data for the Purpose of Evaluating Vaccine Impact
}

\author{
Andrew R Wilson ${ }^{1,}{ }^{*}$, Ryan J. Welch ${ }^{2}$, Mia Hashibe ${ }^{1}$, Jessica Greenwood ${ }^{1}$, Brian Jackson ${ }^{2}$, \\ Rosemary C. She ${ }^{3}$ \\ 1. University of Utah Department of Family and Preventive Medicine, Salt Lake City, Utah \\ 2. ARUP Institute for Clinical and Experimental Pathology, Salt Lake City, Utah \\ 3. Keck School of Medicine of the University of Southern California, Los Angeles, California
}

\begin{abstract}
Nationwide positivity rates of high-risk human papillomavirus for the United States before and since the introduction of a Human Papillomavirus (HPV) vaccine in 2006 would provide insight into the population impact of HPV vaccination. Data for high-risk HPV testing results from January 1, 2004 to June 1, 2013 at a national reference laboratory were retrospectively analyzed to produce 757,761 patient records of women between the ages of 14 and 59. Generalized linear models and finite mixture models were utilized to eliminate sources of bias and establish a population undergoing standard gynecological screening. Unadjusted positivity rates for high-risk HPV were $\mathbf{2 7 . 2 \%}$ for all age groups combined. Highest rates occurred in women aged 14 to 19 . While the positivity rates decreased for all age groups from 2004 to 2013, the higher age categories showed less downward trend following vaccine introduction, and the two age categories 20 to 24 and 25 to 29 showed a significantly different downward trend between pre- and post-vaccine time periods $(-0.1 \%$ per year to $-1.5 \%$ per year, and $0.4 \%$ per year to $-1.5 \%$ per year, respectively). All other age groups had rates of change that became less negative, indicating a slower rate of decline.

Keywords: Human Papillomavirus, Surveillance, Vaccine

Abbreviations: HPV, human papillomavirus; US, United States; FDA, Food and Drug Administration; ACIP, Advisory Committee on Immunization Practices; Pap, Papanicolaou; GLM, generalized linear models; FMM, finite mixture models; $\mathrm{Cl}$, confidence interval; $\mathrm{CDC}$, Centers for Disease Control and Prevention

Correspondence: Email: arw2@utah.edu*

DOI: $10.5210 / o j p h i . v 6 i 3.5593$

Copyright @2014 the author(s)

This is an Open Access article. Authors own copyright of their articles appearing in the Online Journal of Public Health Informatics. Readers may copy articles without permission of the copyright owner(s), as long as the author and OJPHI are acknowledged in the copy and the copy is used for educational, not-for-profit purposes.
\end{abstract}

\section{Introduction}

Numerous studies have demonstrated a clear causal relationship between human papillomavirus (HPV) and cervical cancer, with HPV considered necessary but not sufficient to cause cervical cancer [1-7]. HPV also plays a causative role in vaginal, anal, head, and neck cancers [2,8-10]. 
HPV includes over 100 subtypes and is divided into high- and low-risk groups according to oncogenic risk. In 2006, a quadrivalent vaccine (Gardasil@, Merck) offering protection against high-risk types 16 and 18, and low-risk types 6 and 11, was approved by the Food and Drug Administration (FDA). Additionally, a bivalent vaccine (Cervarix ${ }^{\circledR}$, GlaxoSmithKline) protecting against high-risk types 16 and 18, was approved in 2009 [11]. Both vaccines have shown close to $100 \%$ efficacy against HPV types 16 and 18, the cause of $70 \%$ of all cervical cancers [12]. In June 2006, the Advisory Committee on Immunization Practices (ACIP) recommended vaccination for females 9 to 26 years old [13]. It is hypothesized that with good vaccination coverage, the prevalence of HPV and HPV-associated cancers will decline [14].

Although comprehensive surveillance for genital HPV positivity and prevalence data are considered difficult to estimate, several approximations exist [15]. A 2007 report showed that the overall prevalence in the United States of any HPV infection prior to vaccine introduction (20032004) was approximately $27 \%$ [16]. A follow-up study published in 2013 showed the overall rate from 2007-2010 was 40\% [17]. Both of these studies estimate national level prevalence, but were conducted on limited sample sizes (less than 5000 per time period), and had conflicting rates, with the 2013 study reporting a prevalence during the years 2003-2006 of 43\% [17]. Additional prevalence estimates exist, but these are often targeted at specific populations, lack sufficient sample size, are geographically isolated and often non-U.S. based [18-24]. Therefore, a need exists for surveillance with complete U.S. coverage to establish overall positivity and prevalence rates as well as trends in these rates [12,25].

The goal of this study is to estimate the positivity of high-risk HPV in the United States from 2004 to 2013 using retrospective data from HPV testing conducted at a national reference laboratory. These positivity rates and trends over time should reflect underlying prevalence rates in the population of women who undergo regular gynecological testing and should be useful in supplementing other nationwide estimates of HPV vaccine impact.

This study will illustrate that using such data can overcome the limitations of previous studies because the number of unique patients tested is large and account for a wide geographical spread. Additionally, since HPV testing is typically performed in conjunction with routine Papanicolaou (Pap) testing, and rates of routine testing are above $80 \%$ in U.S. women over 18 years of age, the data represent generalizable rates free from selection bias associated with testing typically performed to support clinical suspicion of disease [26].

\section{Methods}

\section{Study Population and Analysis Datasets}

This study was approved by an Institutional Review Board. Samples are submitted to and tested by a National Reference Laboratory for high-risk HPV (genotypes 16, 18, 31, 33, 35, 39, 45, 51, $52,56,58,59$, and 68). Data from patient test results archived in an electronic data warehouse were extracted for HPV results from January 1, 2004 to June 1, 2013 to produce 757,761 female patient records with conclusive positive or negative results. Attached to each record were test results and demographic data including age, sex, and client information. Using only the first observation for each patient per calendar year between 2004 and 2013, a longitudinal dataset was created, consisting of 735,437 total high-risk HPV results from 590,036 unique patients at 692 unique client sites in 48 U.S. states. 


\section{HPV testing}

Liquid-based endocervical samples were collected and submitted to the National Reference Laboratory for HPV testing. Acceptable sample types include Digene ${ }^{\circledR}$ Cervical Brushes (Qiagen, Hilden, Germany), ThinPrep ${ }^{\circledR}$ PreservCyt ${ }^{\circledR}$ media (Hologic, Inc., Marlborough, MA), and SurePath ${ }^{\mathrm{TM}}$ preservative (Becton-Dickinson, Franklin Lakes, NJ). Testing for HPV was performed according to manufacturer's instructions by the Digene ${ }^{\circledR}$ hc2 HPV DNA Test, which utilized Hybrid Capture 2 technology. ThinPrep ${ }^{\circledR}$ PreservCyt ${ }^{\circledR}$ samples were prepared using the Digene ${ }^{\circledR}$ HC2 Sample Conversion Kit.

\section{Statistical analysis}

Positivity rates were analyzed by age category and year (both individual year and year categories: 2004-2007, 2007-2013) and compared. Frequency tables and exact binomial confidence intervals were constructed to present positivity rates (with 95\% confidence intervals [CI]) by age category and year. Generalized linear models (GLM) were created to assess differences in positivity between age categories and assess changes over time [27]. Two- and three-way interaction models were used to assess both how rates have changed in the pre- and post-vaccine eras and also how age category affects this pre- and post-vaccine era effect on rate, respectively.

To account for potential bias associated with differences in ordering, several methods were employed. First, it was hypothesized that a positive bias might be associated with physicians who submit specimens for HPV testing based on abnormal cytology results [28]. Therefore, finite mixture models were then used to investigate patterns within the distribution of time between sample collection and time of final result release for all patients (Figure 1) [29,30]. Positivity rates between these different testing patterns were compared. Additionally, sensitivity analyses were performed using GLM to assess the effects of time between visit, client size, and client consistency on positivity rates [27]. Time between visits was calculated for patients with more than one visit as the average time between visits. Client size was calculated as number of tests ordered overall and for each year. Client consistency was an indicator variable representing whether or not a client had ordered tests both at the beginning and end of the study period (tests ordered in 2004 and 2013). These derived variables were compared for both their main effect on positivity rate and interaction effects with time on positivity rates.

All calculations were performed using SAS software (v9.3, SAS Institute Inc., Cary, NC, USA). Results were considered statistically significant if $p<0.05$.

\section{Results}

\section{Raw Positivity Rates: High-risk HPV}

Positivity rates for high-risk HPV were separated and compared across time and by age groups (Table 1).

\begin{tabular}{|c|c|c|c|c|c|c|c|c|}
\hline \multirow[b]{2}{*}{$\begin{array}{l}\text { Age, } \\
\text { years }\end{array}$} & \multicolumn{2}{|c|}{ 2004-2013 } & \multicolumn{2}{|c|}{ 2004-2006 } & \multicolumn{2}{|c|}{$2007-2010$} & \multicolumn{2}{|c|}{ 2011-2013 } \\
\hline & No. & $\begin{array}{l}\text { positivity, } \\
\%\end{array}$ & No. & $\begin{array}{l}\text { positivity, } \\
\%\end{array}$ & No. & $\begin{array}{l}\text { positivity, } \\
\%\end{array}$ & No. & $\begin{array}{l}\text { positiv } \\
\%\end{array}$ \\
\hline vera & & 19.4 & & 31.6 & & 18.6 & & 13.2 \\
\hline $14-19$ & 10,979 & 50.6 & 4,692 & 55.5 & 5,224 & 47.8 & 1,063 & 43.0 \\
\hline
\end{tabular}




$\begin{array}{lllllllll}20-24 & 25,725 & 50.2 & 9,276 & 54.1 & 11,525 & 49.5 & 4,924 & 44.4 \\ 25-29 & 24,363 & 36.8 & 7,545 & 41.2 & 11,219 & 36.8 & 5,599 & 30.8 \\ 30-39 & 77,928 & 15.7 & 12,439 & 24.4 & 36,104 & 15.1 & 29,385 & 12.7 \\ 40-49 & 67,841 & 9.5 & 10,824 & 14.7 & 31,955 & 9.0 & 25,062 & 8.0 \\ 50-59 & 49,847 & 7.4 & 6,304 & 12.4 & 23,869 & 7.1 & 19,674 & 6.2\end{array}$

Table 1. Positivity of High-risk HPV According to Age and Year Group in Pattern 1 Individuals from a National Reference Laboratory.

Overall the positivity rate in women aged 14 to 59 years from 2004 to $2013(n=735,437)$ was $27.2 \%$ (95\% confidence interval [CI], 27.1 to 27.3 ). When separated by time period, the positivity rates decreased over time, with the pre-vaccine introduction period (2004 to 2006) having an overall positivity rate of $35.3 \%$ (95\% CI, 35.1 to 35.5) and the final time period (2011 to 2013 ) having an overall positivity rate of $19.7 \%$ (95\% CI, 19.5 to 19.9 ).

When separated by age group, each showed a significant decline in overall positivity over time. The largest absolute decrease was in the 30 to 39 year old age group, with a decrease from $27.2 \%$ (95\% CI, 26.7 to 27.6), in the years 2004 to 2006, to $16.3 \%$ (95\% CI, 16.1 to 16.6), in the years 2011 to 2013. The smallest absolute decrease was in the 25 to 29 year old age group, with a decrease from $44.0 \%$ (95\% CI, 43.4 to 44.6), in the years 2004 to 2006, to $42.7 \%$ (95\% CI, 41.9 to 43.4), in the years 2011 to 2013 (Table 1). Overall, current rates were highest in both the 14 to 19 year old and 20 to 24 year old age categories, with the positivity rate being $54.5 \%$ (95\% CI, 52.9 to 56.2 ), and $54.7 \%$ (95\% CI, 54.0 to 55.5), respectively in the years 2011 to 2013 .

\section{Factors and Significance of Bias}

To establish if physician ordering practices influenced positivity, it needed to be determined if an indication for ordering HPV testing was present prior to sample submission. This was accomplished by analysis of the difference in collection time (reported by the client) and the result time (time at which results are reported from the National Reference Laboratory). Finite mixture models were used and established two distinct populations of patient samples submitted; a population with a peak resulting time minus collection time at three days, and another at eight days (Figure 1).

A nadir (antimode) in the mixture model was observed at five days, which was then used as the cutoff between pattern 1 datasets ( $<5$ days) and pattern 2 datasets ( $>5$ days). This is consistent with previous studies conducted at the National Reference Laboratory and matches data that the majority of cytological results on Pap specimens are completed within five days [28,31]. We also examined the subset of cases with both HPV test and Pap smear results to validate our assumption that HPV testing delayed beyond five days was likely the result of abnormal cytology results. This subset $(n=9,347)$ showed a bimodal peak, with normal cytology results associated with HPV test results on average six days later, whereas abnormal cytology results had HPV results reported on average eight days later. Therefore, it is hypothesized that samples in the pattern 2 dataset were submitted with suspicion of HPV as a result of abnormal cytological findings and should be excluded from our estimates of HPV prevalence. Positivity rates support this, as they are significantly higher in pattern 2 compared to pattern 1 in all age groups across all years (Tables 1, 2). Additionally, sensitivity analyses performed using general linear models showed no statistically significant effect of time between visit, client size, and client ordering consistency on positivity rates. 


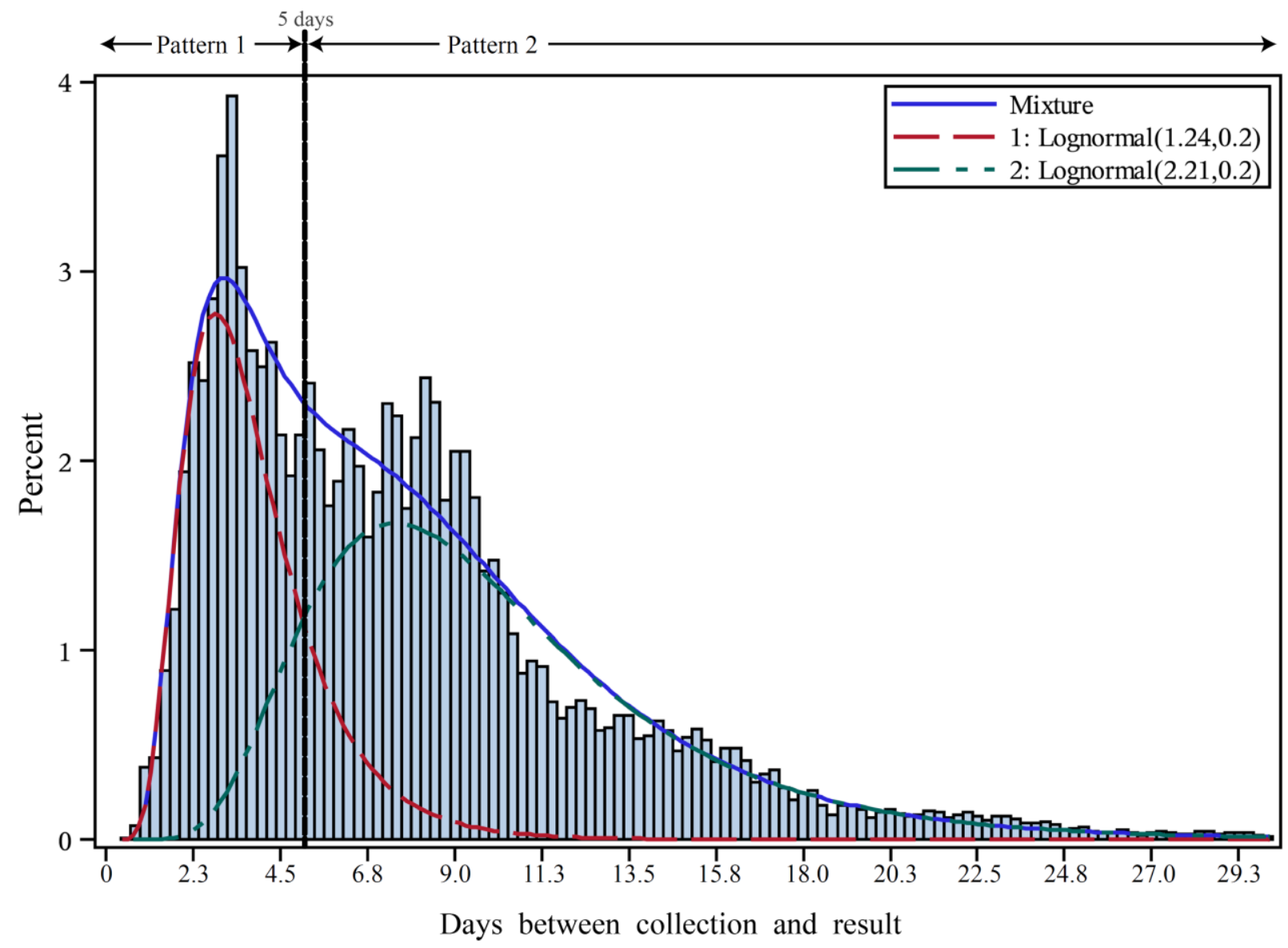

Figure 1: Finite Mixture Modeling (FMM) of time between collection and final result indicating distinct distributions.

\begin{tabular}{llll} 
& \multicolumn{2}{l}{ Rate of positive change per year, \% } & \\
\cline { 2 - 3 } $\begin{array}{l}\text { Age, } \\
\text { years }\end{array}$ & $2004-2006$ & $2007-2013$ & Difference \\
\hline $14-19$ & -2.2 & -1.5 & 0.7 \\
$20-24$ & -0.1 & -1.5 & $-1.4^{*}$ \\
$25-29$ & 0.4 & -1.5 & $-1.9^{*}$ \\
$30-39$ & -3.0 & -0.8 & $2.2^{*}$ \\
$40-49$ & -1.7 & -0.4 & $1.3^{*}$ \\
$50-59$ & -1.4 & -0.3 & $1.1^{*}$ \\
\hline
\end{tabular}

Table 2. Average Rate of Change is Positivity Per Year Comparing Pre- and Post-Vaccine Periods.

${ }^{\mathrm{a}} \mathrm{P}<0.05$

\section{Trends: High-risk HPV}

Following investigation of potential influences on the positivity rate and determining if they had significance or not, it was estimated that the positivity rates in pattern 1 should provide a useful indicator of underlying population prevalence of sexually active women getting regular 
gynecological screening. Overall positivity in this pattern 1 group of high-risk HPV for women aged 14 to 59 years from 2004 to 2013 was $19.4 \%$ (95\% CI, 19.3 to 19.6). Over time, the positivity rates decreased from $31.6 \%$ (95\% CI, 31.2 to 32.0) during the years 2004 to 2006, to $13.2 \%$ (95\% CI, 13.0 to 13.4) during the years 2011 to 2013.

Women aged 14 to 19 years showed the largest absolute decrease in the pattern 1 group in positivity from $55.5 \%$ (95\% CI, 54.0 to 56.9 ) during the years 2004 to 2006 , to $43.0 \%$ (95\% CI, 40.0 to 45.9 ) during the years 2011 to 2013 . Women aged 50 to 59 showed the largest percent decrease, dropping $49.7 \%$ in prevalence from $12.4 \%$ (95\% CI, 11.6 to 13.2) during the years 2004 to 2006 , to $6.2 \%$ (95\% CI, 5.9 to 6.6) during the years 2011 to 2013 . The smallest percent decrease was seen in women aged 20 to 24 , with only an $18.0 \%$ reduction in positivity from $54.1 \%$ (95\% CI, 53.1 to 55.1) during the years 2004 to 2006, to $44.4 \%$ (95\% CI, 43.0 to 45.8 ) during the years 2011 to 2013.

Rates of change in high-risk HPV positivity per year were calculated and compared between the pre-vaccination (2004-2006) and post-vaccination periods (2007-2013) in all age groups. Generalized linear models showed that age category had a significant effect on rates when these time periods were compared. In the pattern 1 group, all age categories showed positivity decreases in the post-vaccine period; however, only women aged 20 to 24 and 25 to 29 showed negative differences $(-1.4 \%$ per year and $-1.9 \%$ per year, respectively) when pre- and postvaccine period rates of change were compared. All other age categories had rates of change that were less negative, and closer to zero (Figure 2, Table 2).

\section{Discussion}

The goal of this study was to establish and evaluate a tool to estimate the current positivity of and trends in high-risk HPV in women in the United States from 2004 to 2013; specifically, in women who undergo regular gynecological screenings. These data indicate that the overall positivity of HPV is declining, especially in young women; however, the rate at which positivity is declining is slower than other studies indicate in certain age categories [17]. This may be a combination of insufficient vaccination coverage as well as these data being a mixture of vaccine-preventable and other high-risk types collectively. Mathematical models have predicted that the introduction of the vaccine should have a strong impact on HPV positivity rates in the types covered by the vaccine [14]. While this data could not be separated entirely by type, a reduction of 18 to $25 \%$ in positivity in all high-risk HPV was seen in women aged 14 to 29 when comparing rates prior to the vaccine introduction (2004 to 2006) to current rates (2011 to 2013).

While most studies performed to determine HPV positivity or prevalence rely on survey-based methods, this study has several strengths that allow for the generalizability of the results to women who undergo regular gynecological screening in the US. First, the data were retrospective data from a large national reference laboratory, which created a large dataset of more than 700,000 high-risk HPV results that accurately reflected at-risk and vaccine-targeted population. Second, the data were filtered in several ways: only the first visit per calendar year of each patient was used to reduce redundancy that may occur as a result of repeat confirmatory testing, and potential ordering bias was reduced by separating results from patient samples believed to be submitted because of abnormal cytology results. To account for ordering bias, finite mixture modeling was used to determine where, if any, a separation may exist between the time the samples were collected and the time that results were entered [29]. The separation 
observed is likely the result of samples being immediately sent for HPV testing versus samples that had been

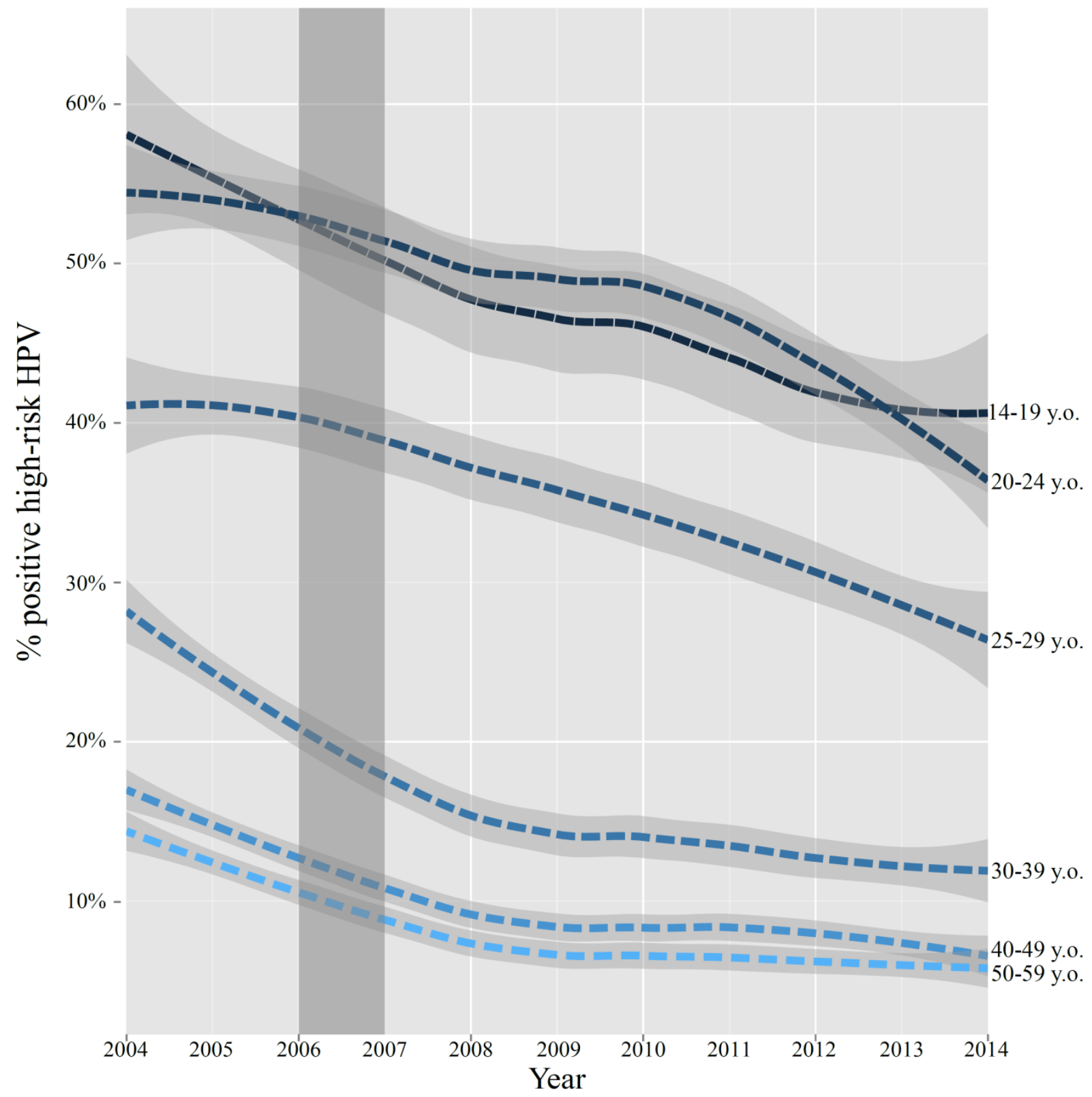

Figure 2: High-risk HPV positivity in pattern 1 individuals by year and age category from a National Reference Laboratory.

screened for abnormal cytology before being sent for HPV testing [28]. The cutoff between pattern 1 and 2 of five days can be further supported by a 2013 survey by the College of American Pathologists (CAP) that showed that $83.9 \%$ of Papanicolaou testing took less than five days to completion, with the majority taking 3 to 4 days [31]. Additionally, the positivity rates in testing pattern 2 were significantly higher than in testing pattern 1, suggesting physicians had an 
indication for ordering HPV testing, such as abnormal Pap smear results. Lastly, sensitivity analyses were performed to determine if differences in clients might influence the positivity rates. Since these data included many individual client sites, it was theorized that differences in client size might account for a bias in positivity: clients with fewer sample submissions may be targeting at-risk individuals with a higher likelihood of disease. Using generalized linear models, it was shown that there were no differences in positivity rates when adjusting for client size, location, or ordering trends, further supporting that the positivity rates truly are indicative of overall prevalence.

Based on retrospective data from a national reference lab from 2004 to 2013, the overall positivity of high-risk HPV in all age groups was $27.2 \%$. Looking at only pattern 1 , which was established as an estimate of unbiased prevalence, the rate drops to $19.4 \%$. Our estimate of prevaccine high-risk HPV positivity in women 14 to 59 years old was $31.6 \%$, compared to the rates reported from other studies of $15-29 \%$ [16,17]. For the current time period (2010-2013), the positivity rate decreases to $13.2 \%$ for all women aged 14 to 59 . By age group, our positivity estimates are also higher, particularly in the 14-19 year old group (55-57\%) when compared to CDC data (15-20\%). This could be due to the fact that HPV testing is generally only performed for sexually active women; so positivity estimates in this study likely reflect the positivity rates in the sexually active population, which has been shown to be close to 50\% in young adult females, similar to the present study [16,17]. Furthermore, the method of specimen collection differed from other studies in that provider-collected cervical swabs were used as opposed to self-collected cervicovaginal swabs. Provider collection could not feasibly be standardized in this study, but overall performance of HPV testing has been shown to be similar for both collection methods [32].

It is important to note that despite a decline in high-risk HPV positivity in all age categories, the rate of positivity change per year is not consistent. HPV vaccination is only recommended in women up to the age of 26; therefore, this group would likely have a faster decrease in positivity. It could further be postulated that, since the vaccinated population is increasing in age, an inverse relationship between age and change in prevalence per year would exist. This was observed, with the post-vaccine introduction period rates of positivity change per year being consistent in the vaccine target group (age groups 14 to 19, 20 to 24 , and 25 to 29 ) at a $-1.5 \%$ and decreasing with each increase in age category to a low of $-0.3 \%$ in the 50 to 59 year old group. The decreasing rates that were seen in the pre-vaccine introduction period are likely due to inconsistent testing practices among physicians and an increasingly heterogeneous population being screened; however, these rates still provide a baseline to compare post-vaccine period rates. Crucially, the difference in the pre- and post-vaccine era rates of positivity change were only more negative in the age groups that had overlap in vaccine and screening guidelines (20 to 24 and 25 to 29 -yearold women). This suggests that the decreases in the rates of prevalence change per year seen in the younger population age categories could be due to increasing vaccination rates.

For comparison, 618,261 Chlamydia tests were analyzed for females stratified by the same age categories as for HPV and covering years 2004-2013 (Supplemental online figure). Across all age categories, there is no significant downward trend in positivity rates and, in fact, most age categories show an upward trend (especially since 2008). These results further support the hypothesis that downward trends observed in HPV are likely attributable to vaccine uptake. 


\section{Limitations of Study}

Several limitations to this study exist. Current guidelines recommend screening every three years in all sexually active women over 21 years of age; therefore, and as a result of the data being collected at a national reference lab, the population represented is likely a sexually active population with access to healthcare. Furthermore, cervical cancer screening guidelines have changed over the duration of data collection, with the introduction of co-testing HPV and Pap screening in women older than 30 years and recommendation against screening in women less than 21 years regardless of first sexual contact $[33,34]$. Despite these changes, these data and several studies indicate that the guidelines are not being followed as testing is still frequently performed on an annual basis, in women under 21 years of age, and in women following hysterectomy $[28,35,36]$. Another final limitation is that the Hybrid Capture 2 method does not differentiate genotypes and differentiation of specific high-risk vaccine preventable HPV strain was not possible [37,38].

We attempted to mitigate some of the potential biases by focusing on collection pattern 1 (results within five days of collection) and only including one visit within the calendar year, but future studies of this kind will certainly be needed to both validate and improve upon methodology. Additional studies that have access to multiple testing sites may be able to refine analyses by using characteristics of the different client sites: for example, differences in ordering volumes, within-center positivity rates, or information collected outside of the analytic framework, such as clinical indications for testing in their population. Our study demonstrates the potential for using HPV test data from large national reference laboratories to supplement the ongoing and planned efforts to monitor HPV vaccine impact in the US [39].

\section{Conclusion}

In evaluating the surveillance tool, we find that it is important to consider many sources of heterogeneity, e.g., age, type of test, location, and type of testing center, and also consider quantitative methods of adjustment and distribution assessment to construct a useful surveillance tool. Further studies should expand on this methodology.

The results of the surveillance tool indicate a downward trend in vaccine-appropriate age groups consistent with uptake of the HPV vaccine. The pre-post rate changes were in direct contrast between the age-appropriate groups and the groups too old for the HPV vaccine, further indicating the surveillance tool may be detecting the impact of the HPV vaccine over time. After refinement, this surveillance tool should remain in place to observe the future impact of the HPV vaccine.

\section{Acknowledgements}

The authors would like to thank David Davis of ARUP laboratories for his help acquiring the data used in this study. The authors would also like to thank Lindsay Larkin Wilson for her help in editing the manuscript.

\section{References}

1. Lowy DR, Schiller JT. 2006. Prophylactic human papillomavirus vaccines. J Clin Invest. 116(5), 1167-73. PubMed http://dx.doi.org/10.1172/JCI28607 
2. WHO/ICO Information Centre on Hpv and Cervical Cancer. (hpv Information Centre). Human Papillomavirus and Related Cancers in United States of America. 2010.

3. McIntosh J, Sturpe DA, Khanna N. Human papillomavirus vaccine and cervical cancer prevention: practice and policy implications for pharmacists. J Am Pharm Assoc JAPhA. 2008 Feb;48(1):e1-13; quiz e14-17.

4. Doorbar J, Quint W, Banks L, Bravo IG, Stoler M, et al. 2012. The biology and life-cycle of human papillomaviruses. Vaccine. 30(Suppl 5), F55-70. PubMed http://dx.doi.org/10.1016/j.vaccine.2012.06.083

5. Bosch FX, Manos MM, Muñoz N, Sherman M, Jansen AM, et al. 1995. Prevalence of human papillomavirus in cervical cancer: a worldwide perspective. International biological study on cervical cancer (IBSCC) Study Group. J Natl Cancer Inst. 87(11), 796-802. PubMed http://dx.doi.org/10.1093/jnci/87.11.796

6. Walboomers JM, Jacobs MV, Manos MM, Bosch FX, Kummer JA, et al. 1999. Human papillomavirus is a necessary cause of invasive cervical cancer worldwide. J Pathol. 189(1), 12-19. PubMed http://dx.doi.org/10.1002/(SICI)1096-9896(199909)189:1<12::AIDPATH431>3.0.CO;2-F

7. Song S, Pitot HC, Lambert PF. 1999. The human papillomavirus type 16 E6 gene alone is sufficient to induce carcinomas in transgenic animals. $J$ Virol. 73(7), 5887-93. PubMed

8. Gillison ML, Lowy DR. 2004. A causal role for human papillomavirus in head and neck cancer. Lancet. 363(9420), 1488-89. PubMed http://dx.doi.org/10.1016/S0140$\underline{6736(04) 16194-1}$

9. Fakhry C, Gillison ML. 2006. Clinical implications of human papillomavirus in head and neck cancers. $J$ Clin Oncol. 24(17), 2606-11. PubMed http://dx.doi.org/10.1200/JCO.2006.06.1291

10. Zandberg DP, Bhargava R, Badin S, Cullen KJ. 2013. The role of human papillomavirus in nongenital cancers. CA Cancer $J$ Clin. 63(1), 57-81. PubMed http://dx.doi.org/10.3322/caac.21167

11. Bouvard V, Baan R, Straif K, Grosse Y, Secretan B, et al. 2009. A review of human carcinogens--Part B: biological agents. Lancet Oncol. 10(4), 321-22. PubMed http://dx.doi.org/10.1016/S1470-2045(09)70096-8

12. Centers for Disease Control and Prevention (CDC). 2012. Human papillomavirus-associated cancers - United States, 2004-2008. MMWR Morb Mortal Wkly Rep. 61, 258-61. PubMed

13. Markowitz LE, Dunne EF, Saraiya M, Lawson HW, Chesson H, et al. 2007. Quadrivalent Human Papillomavirus Vaccine: Recommendations of the Advisory Committee on Immunization Practices (ACIP). MMWR Recomm Rep. 56(RR-2), 1-24. PubMed

14. Barnabas RV, Laukkanen P, Koskela P, Kontula O, Lehtinen M, et al. 2006. Epidemiology of HPV 16 and cervical cancer in Finland and the potential impact of vaccination: mathematical modelling analyses. PLoS Med. 3(5), e138. PubMed http://dx.doi.org/10.1371/journal.pmed.0030138

15. Satterwhite CL, Torrone E, Meites E, Dunne EF, Mahajan R, et al. 2013. Sexually transmitted infections among US women and men: prevalence and incidence estimates, 
2008. Sex Transm Dis. 40(3), 187-93. $\quad$ PubMed http://dx.doi.org/10.1097/OLQ.0b013e318286bb53

16. Dunne EF, Unger ER, Sternberg M, McQuillan G, Swan DC, et al. 2007. Prevalence of HPV infection among females in the United States. JAMA. 297(8), 813-19. PubMed http://dx.doi.org/10.1001/jama.297.8.813

17. Markowitz LE, Hariri S, Lin C, Dunne EF, Steinau M, et al. 2013. Reduction in human papillomavirus (HPV) prevalence among young women following HPV vaccine introduction in the United States, National Health and Nutrition Examination Surveys, 20032010. J Infect Dis. 208(3), 385-93. PubMed http://dx.doi.org/10.1093/infdis/jit192

18. Banister CE, Messersmith AR, Chakraborty H, Wang Y, Spiryda LB, et al. 2013. HPV prevalence at enrollment and baseline results from the Carolina Women's Care Study, a longitudinal study of HPV persistence in women of college age. Int J Womens Health. 5, 379-88. PubMed

19. Reiter PL, Katz ML, Ruffin MT, Hade EM, DeGraffenreid CR, et al. 2013. HPV prevalence among women from Appalachia: results from the CARE project. PLOS ONE. 8(8), e 74276. PubMed http://dx.doi.org/10.1371/journal.pone.0074276

20. Reiter PL, Katz ML, Ruffin MT, Hade EM, Degraffenreid CR, et al. 2013. Correction: HPV Prevalence among Women from Appalachia: Results from the CARE Project. PLoS ONE. 8(10). http://dx.doi.org/10.1371/annotation/7a4cfd0a-cf77-430e-89a0-b2576268e2eb

21. Leinonen MK, Anttila A, Malila N, Dillner J, Forslund O, et al. 2013. Type- and agespecific distribution of human papillomavirus in women attending cervical cancer screening in Finland. Br J Cancer. 109(11), 2941-50. PubMed http://dx.doi.org/10.1038/bjc.2013.647

22. Walmer DK, Eder PS, Bell L, Salim H, Kobayashi L, et al. 2013. Human papillomavirus prevalence in a population of women living in Port-au-Prince and Leogane, Haiti. PLoS ONE. 8(10), e76110. PubMed http://dx.doi.org/10.1371/journal.pone.0076110

23. Dunne EF, Klein NP, Naleway AL, Baxter R, Weinmann S, et al. 2013. Prevalence of HPV types in cervical specimens from an integrated healthcare delivery system: baseline assessment to measure HPV vaccine impact. Cancer Causes Control. 24(2), 403-07. PubMed http://dx.doi.org/10.1007/s10552-012-0085-9

24. Škamperle M, Kocjan BJ, Maver PJ, Seme K, Poljak M. 2013. Human papillomavirus (HPV) prevalence and HPV type distribution in cervical, vulvar, and anal cancers in central and eastern Europe. Acta Dermatovenerol Alp Pannonica Adriat. 22(1), 1-5. PubMed

25. Wilson A, Welch R, She R. Surveillance of Human Papilloma Virus in the United States to Evaluate Vaccine Impact. Online J Public Health Inform [Internet]. 2014 Mar 9 [cited 2014 Apr 30];6(1). Available from: http://ojphi.org/ojs/index.php/ojphi/article/view/5172

26. Behavioral Risk Factor Surveillance System [Internet]. Centers for Disease Control and Prevention. Available from: http://www.cdc.gov/brfss/about/about_brfss.htm

27. McCullagh P, Nelder JA. Generalized Linear Models, Second Edition. 2 edition. Boca Raton: Chapman and Hall/CRC; 1989. 532 p. 
28. Shirts BH, Jackson BR. 2010. Informatics methods for laboratory evaluation of HPV ordering patterns with an example from a nationwide sample in the United States, 20032009. J Pathol Inform. 1, 26. PubMed http://dx.doi.org/10.4103/2153-3539.73504

29. Day NE. 1969. Estimating the components of a mixture of normal distributions. Biometrika. 56(3), 463-74. http://dx.doi.org/10.1093/biomet/56.3.463

30. McLachlan G, Peel D. Finite Mixture Models. 1 edition. New York: Wiley-Interscience; 2000. $456 \mathrm{p}$.

31. Clary KM, Davey DD, Naryshkin S, Austin RM, Thomas N, et al. 2013. The role of monitoring interpretive rates, concordance between cytotechnologist and pathologist interpretations before sign-out, and turnaround time in gynecologic cytology quality assurance: findings from the College of American Pathologists Gynecologic Cytopathology Quality Consensus Conference working group 1. Arch Pathol Lab Med. 137(2), 164-74. PubMed http://dx.doi.org/10.5858/arpa.2012-0120-CC

32. Snijders PJF, Verhoef VMJ, Arbyn M, Ogilvie G, Minozzi S, et al. 2013. High-risk HPV testing on self-sampled versus clinician-collected specimens: a review on the clinical accuracy and impact on population attendance in cervical cancer screening. Int $J$ Cancer. 132(10), 2223-36. PubMed http://dx.doi.org/10.1002/ijc.27790

33. Saslow D, Solomon D, Lawson HW, Killackey M, Kulasingam S, et al. 2012. American Cancer Society, American Society for Colposcopy and Cervical Pathology, and American Society for Clinical Pathology Screening Guidelines for the Prevention and Early Detection of Cervical Cancer. CA Cancer J Clin. 62(3), 147-72. http://dx.doi.org/10.3322/caac.21139

34. Committee on Practice Bulletins-Gynecology. 2012. ACOG Practice Bulletin Number 131: Screening for cervical cancer. Obstet Gynecol. 120(5), 1222-38. PubMed

35. Roland KB, Benard VB, Soman A, Breen N, Kepka D, et al. 2013. Cervical Cancer Screening Among Young Adult Women in the United States. Cancer Epidemiol Biomarkers Prev. 22(4), 580-88. PubMed http://dx.doi.org/10.1158/1055-9965.EPI-12-1266

36. Kepka D, Breen N, King JB, Benard VB, Saraiya M. 2014. Overuse of Papanicolaou Testing Among Older Women and Among Women Without a Cervix. JAMA Intern Med. 174(2), 293-6. PubMed http://dx.doi.org/10.1001/jamainternmed.2013.12607

37. Castle PE, Solomon D, Wheeler CM, Gravitt PE, Wacholder S, et al. 2008. Human papillomavirus genotype specificity of hybrid capture 2. J Clin Microbiol. 46(8), 2595-604. PubMed http://dx.doi.org/10.1128/JCM.00824-08

38. Sargent A, Bailey A, Turner A, Almonte M, Gilham C, et al. 2010. Optimal threshold for a positive hybrid capture 2 test for detection of human papillomavirus: data from the ARTISTIC trial. $J$ Clin Microbiol. 48(2), 554-58. http://dx.doi.org/10.1128/JCM.00896-09

39. Markowitz LE, Hariri S, Unger ER, Saraiya M, Datta SD, et al. 2010. Post-licensure monitoring of HPV vaccine in the United States. Vaccine. 28(30), 4731-37. PubMed http://dx.doi.org/10.1016/j.vaccine.2010.02.019 


\section{Supplementary Material}

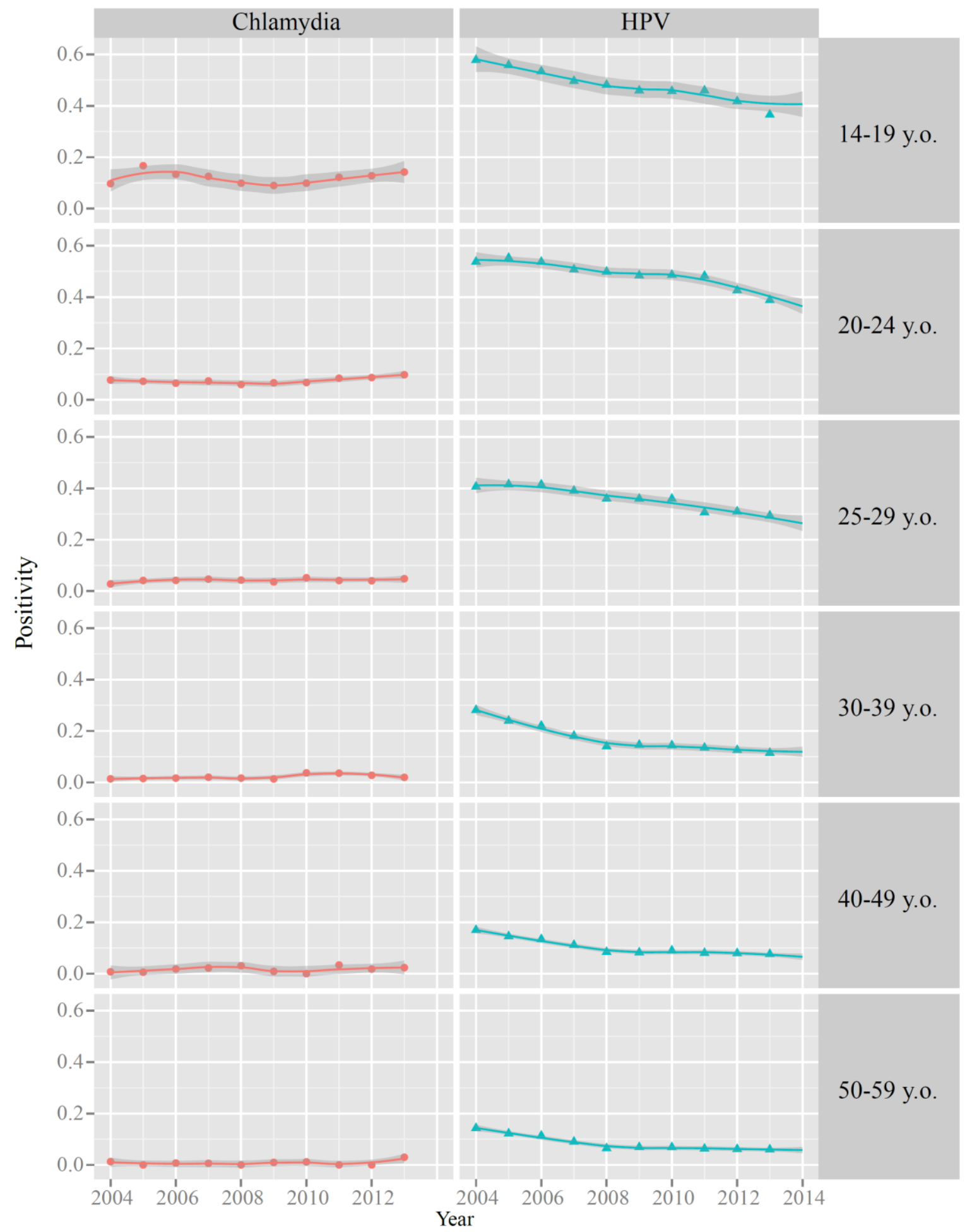

Supplemental figure: Comparison of positivity rates between high-risk HPV (pattern 1 individuals) and Chlamydia by year and age category from a National Reference Laboratory. 